Part 4. Star Cluster Formation and Evolution: Theory and Observation

Section A. Invited Reviews and Contributed Talks 


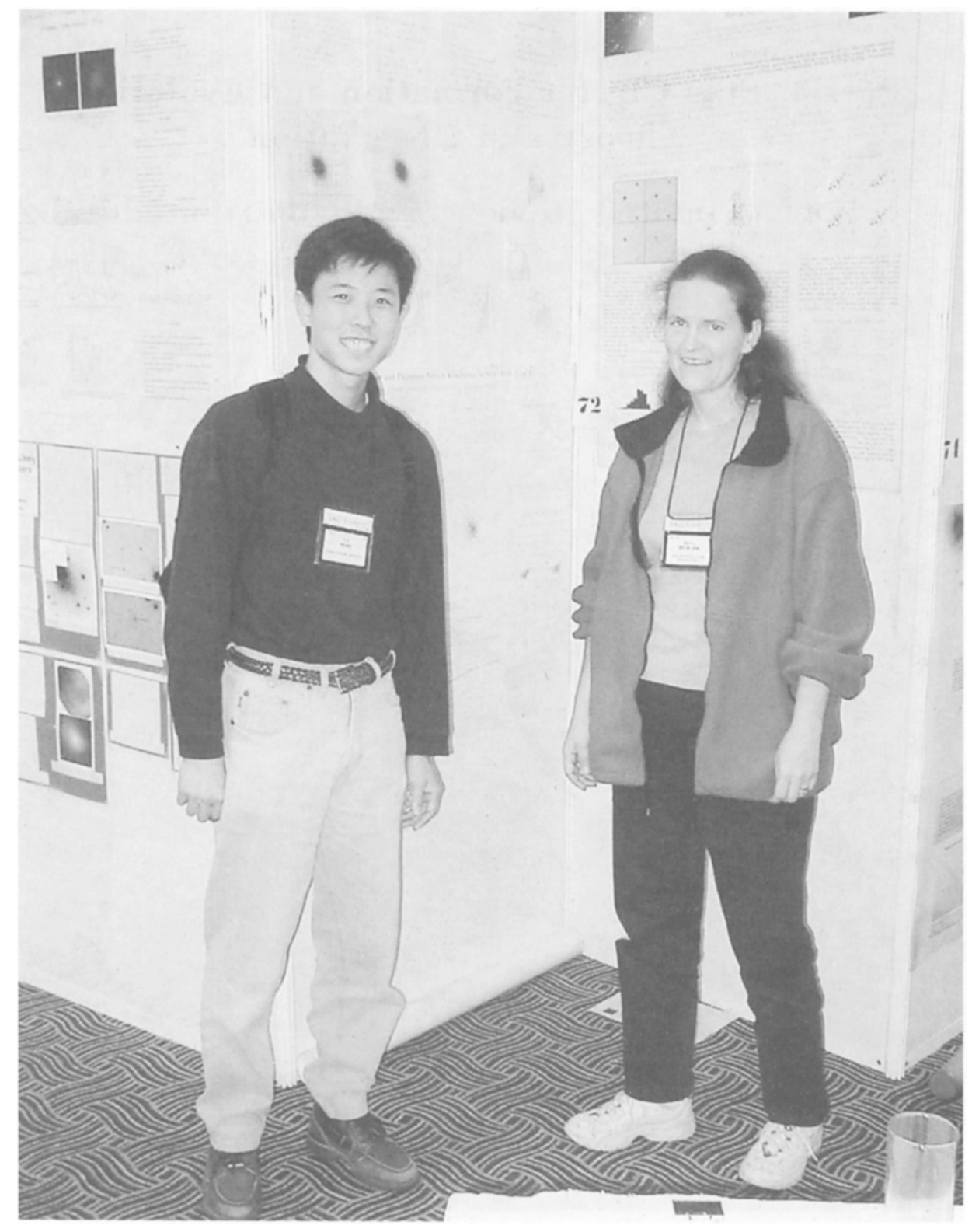

Eric Peng and Marina Rejkuba discuss - what else? globular clusters in NGC 5128. 


\title{
Star Formation in Large N Clusters
}

\author{
C.J. Clarke \\ Institute of Astronomy, Madingley Road,, Cambridge, U.K., CBз OHA
}

\begin{abstract}
We focus here on the gas dynamics of cluster formation and the early stellar dynamical evolution of young clusters. We point out that the condition that a cloud can fragment into a large number of pieces places rather particular constraints on its initial state; we also review the processes that shape the stellar IMF in cluster formation simulations. We show how N-body calculations and observations can be combined to discover the properties of clusters at the point at which they first become stellar dynamical (as opposed to gas dynamical systems). Finally, we touch on the question of how proto-cluster clouds are assembled and reopen the issue of whether dark matter may play a role in globular cluster formation.
\end{abstract}

\section{Introduction}

In this Chapter we review our current understanding of the formation of populous clusters. The fundamental issue here is how a cloud of gas, mass $M_{c}$, is able to split into a large number of pieces, whose mass scale $M_{*}$ is many orders of magnitude less than $M_{c}$. A related question is why $M_{*}$ is apparently roughly the same in all observed systems, whereas the cluster mass scale (considering the range from a few star system to globular clusters) spans more than five orders of magnitude.

Numerical simulations, in fact, find it remarkably hard to achieve this splitting into a large number of pieces, as is required in the formation of a large $N$ cluster. One interpretation is that this is merely an artifact of the numerical technique employed: adequate resolution plays a critical role in ensuring that simulations indeed show fragmentation. Fortunately, this issue has been rather thoroughly explored in the context of less ambitious fragmentation calculations, namely in the formation of binary and multiple star systems (Bate and Burkert 1997). If, as it would seem, this difficulty is not a numerical one, then it leads to a more interesting conclusion - i.e., that particular initial conditions are required for cluster formation and that these conditions are routinely delivered in nature.

In Section 2, we review recent work on the hydrodynamics of collapse and fragmentation and draw preliminary conclusions about the requisite conditions for cluster formation. We stress that although the theoretical issues underlying these simulations have been discussed, and analysed in simple geometries, for decades (e.g., Hoyle 1953, Hunter 1962, Larson 1978, Lubow and Pringle 1993) it is only very recently that it has proved possible to undertake the sort of large 
scale 3D simulations that are required for studying cluster formation. Indeed, the state of the art in this regard is represented by simulations that produce less than a hundred stars. We are thus still well short of being able to perform fully resolved simulations of globular cluster formation, although the insights from these calculations can still be usefully applied to the larger scale problem.

Section 3 explores a 'cleaner', later, stage in the evolution of young clusters, i.e., from the point at which they become mainly stellar dynamical (as opposed to gas dynamical) systems. N-body calculations can be used to map the observed state of young clusters (regarding their shapes, mass distribution and homogeneity for example) back to the point at which the stars became the majority mass component.

Finally, in Section 4 we briefly visit the 'assembly' problem, i.e., the question of how the large masses of gas are brought together prior to the onset of star formation. We attempt no review in this regard, as other chapters will address this issue in more detail . Instead, we report on recent work which suggests that dark matter may play a significant role in gas assembly in the case of globular clusters forming within protogalactic halos.

\section{The Hydrodynamics of Cluster Formation.}

In principle, a cluster of $N$ stars may arise through two routes: the fragmentation of $(<N)$ Jeans unstable gas clumps, or else through the coalescence of $(>N)$ Jeans stable clumps. In this contribution, we do not address in any detail how any clumpiness in the initial conditions is generated, though we note that whereas self-gravity can be invoked in the production of Jeans unstable clumps, in the opposite limit it is necessary to invoke some non-gravitational mechanism. For example, Murray and Lin 1989 have argued that thermal instabilities, associated with cooling by molecular hydrogen, can promote the formation of a two phase medium during the collapse of protoglobular clusters. Such instability leads to the break-up of the gas into sub-Jeans mass cloudlets, so that star formation must then proceed through an agglomerative process. Murray and Lin 1996 (see also Murray et al. 1999) have argued that such a coalescence model produces a roughly power law IMF in the resulting stars.

Recent attempts to reproduce these results using Smoothed Particle Hydrodynamics (SPH) simulations of colliding, sub-Jeans mass, clumps have indicated a very different picture however. In the SPH simulations (Gittins et al., in prep.), the situation after a mean free collision time between two clumps, is that whereas around half the clumps have not suffered a collision yet, most of the remainder are embroiled in one or two regions of collapsed gas surrounded by halos of infalling disrupted clumps. Whereas the simulations require more detailed scrutiny, it would seem that the key difference is that real hydrodynamical clump-clump collisions are more disruptive than those envisaged by Murray and Lin, which essentially result in the incompressible merger of each colliding pair. Such disruptive collisions, together with tidal shredding as massive collapsed regions develop, are probably the reason for the very different outcome, in terms of mass spectrum, of the hydrodynamical simulations. The resulting collapsed regions of course contain a large number of Jeans masses, and thus 
may themselves provide the sites of cluster formation through fragmentation. Henceforth, we focus on this fragmentation scenario for cluster formation.

\subsection{The first cluster formation simulations.}

Advances in computing power have in recent years permitted the first attempts to model cluster formation from first principles (Klessen et al. 1998, Bate et al., in prep.). The computational requirements, even for rather modest clusters, are however awesome. An important aspect of all SPH codes is that they evaluate physical quantities through weighted averaging over regions containing $\sim 50$ particles. Consequently, one should regard a resolution element in SPH as comprising 50 particles: thus in making crude comparisons between the resolution requirements of SPH and Eulerian codes, one should multiply the number of grid points in a finite difference method by 50 to obtain the required number of SPH particles. Furthermore, gravitationally unstable gas will not collapse in a simulation unless the mass resolution is less than a Jeans mass (Bate and Burkert 1997). Naively, one might suppose that this places a minimum resolution requirement of, say, 100 particles per 'star'. The situation is much more demanding than this, however, because stars may form by the gravitational collapse of a region that comprises a mass much less than the ultimate stellar mass, with the remaining mass being subsequently accreted onto the initial collapsed core. In order to ensure that all regions that should collapse will indeed do this in the simulation, it is necessary that the code should assign 50 particles to the minimum Jeans mass that can collapse. For the equation of state employed in SPH calculations this minimum mass, is $\sim 10^{-3} M_{\odot}$. It thus follows that simulations producing only a few tens of stars (typical mass $\sim 1 M_{\odot}$ ) require in excess of $10^{6}$ particles.

Such large particle numbers necessitate the use of supercomputers with massively parallel architecture, such as the U.K. Astrophysical Fluids Facility (UKAFF). Pilot UKAFF simulations of cluster formation by Bate et al. (in prep.) have amply demonstrated the difficulties involved in achieving fragmentation into a large number of stars. In general, it is found that the final number of fragments ('stars') is less than the number of Jeans masses contained in the initial conditions, this latter being a 'rule of thumb' suggested by Larson (1978) on the basis of early fragmentation calculations. A more correct statement would appear to be that the number of stars is approximately equal to the number of spatially connected Jeans masses in the initial conditions contained in nonlinear fluctuations. [Note that this implies that the Rees-Ostriker criterion for fragmentation - that the cooling time should be much less than the dynamical timescale (Rees and Ostriker 1977) - is thus a necessary but not a sufficient criterion for fragmentation: not only must the initial conditions contain a large number of Jeans masses (as would result from faster than dynamical cooling) but the gravitationally unstable gas needs also to be appropriately arranged.]

It would thus seem that achieving large $\mathrm{N}$ fragmentation involves a 'putting in of the answer' in the initial conditions. Since globular clusters are systems that have managed to fragment into an extremely large number of pieces $\left(\sim 10^{6}\right)$, this would suggest that the gas from which globular clusters formed contained a rich non-linear internal structure. Such conditions are readily provided by the observed state of Giant Molecular Clouds (GMCs), which are characterised 
by negligible thermal pressure, supersonic turbulence and resulting strong nonlinear density structure. [See also Padoan et al. 1997, McLaughlin and Pudritz 1996, Harris and Pudritz 1994, Elmegreen and Efremov 1997 for previous advocacy of the view that globular cluster formation involves a scaled up version of the star forming conditions observed in GMCs; a key difference, of course, is that in order to form bound clusters, the star formation efficiency in proto-globular clouds must be one to two orders of magnitude higher than that observed in typical GMCs (Lada et al. 1984, Goodwin 1997)].

On the other hand, unsuitable pre-conditions for large $N$ fragmentation are probably provided by any scenario in which the progenitor cloud is thermally supported and then passes through a phase of quasi-static cooling - the concern here is that the cloud would become too homogenised during this phase, so that it wouldn't contain the requisite density structure for fragmentation, regardless of the rapidity of subsequent cooling. Such arguments would militate against scenarios in which the cluster mass scale is essentially a Jeans mass (such as that of Fall and Rees 1985), since this implies that thermal pressure is competitive with gravity at the stage that the protoglobular cloud is assembled. Instead, one should perhaps seek scenarios in which the protoglobular cloud is assembled in a highly Jeans unstable condition (see Section 4).

The 'successful' simulations of Bate et al. (see also Klessen et al. 1998) involve initial conditions that are both cold and are either highly inhomogeneous or else contain a supersonic turbulent velocity field that rapidly generates such inhomogeneities. Typically, the densest regions collapse first and then grow in mass due to subsequent accretion from distributed lower density gas. A complex web of knotty filamentary structures develop, in which cores formed at early times 'compete' for the available mass reservoir. The mass spectrum of the resulting cores is approximately Salpeter. Below we dissect the predictions of the competitive accretion scenario in more detail.

\subsection{Competitive accretion in detail}

Considerable insight into how the competitive accretion process shapes the resulting stellar mass spectrum has been provided by idealised hydrodynamical simulations (Bonnell et al. 2001a), which replace the complexity of the simulations described above by a simple 'two tier' structure - i.e., the initial density structure is provided by a mixture of already collapsed 'seeds' and a smooth background containing the majority of the mass. Bonnell et al. 2001b) approximately characterised the accretion histories of 'seeds' in different regions of the cluster and thereby derived analytic expressions for the resulting IMF. It turns out that the IMF can be approximated by two power laws, with the steeper one at higher masses. These two power laws represent the two regimes in which stars acquire their mass. Near the cluster core, where the stars soon dominate over the gas, the stars virialise and have a high velocity relative to residual inflowing gas. The appropriate accretion cross-section is then the Bondi Hoyle one, and the slope of the resulting IMF is similar to Salpeter (in the range $2-2.5$ ) as originally suggested by Zinnecker (1982). At larger radii, the gas dominates the potential; here the seeds and gas collapse together so that their relative velocities, $v_{r e l}$, are low. The resulting Bondi Hoyle radii of the seeds (which scale as $\left.v_{r e l}^{-2}\right)$ would then be so large that the accretion flow at this impact parameter 
would be disrupted by the tidal field of the cluster. If the seeds' tidal radii are used instead in defining the accretion cross section, one may show that the resulting IMF is a power law of slope 1.5. Thus competitive accretion naturally produces a two power law IMF (flatter at low mass), with the regimes separated by whether stars have accreted the majority of their mass in star dominated or gas dominated regimes.

This competitive accretion process naturally sets up a cluster that is mass segregated at birth; the above analytic arguments lead to a rather tight correspondence between stellar mass and radius in the cluster. However, in hydrodynamical simulations of this 'two tier' situation, the correspondence between ultimate stellar mass and radius in the cluster is rather blurred by the stochastic nature of cluster dynamics (Bonnell et al. 2001a): in practice, whereas low mass stars are formed at all radii, the highest mass stars are formed preferentially in the middle (see 3.3 below).

Although the mass spectra produced in the 'realistic' simulations described in 2.1 above have yet to be analysed in detail, the overall similarity between the resultant spectrum in this case and in these idealised simulations may point to the importance of competitive accretion in turbulent fragmentation calculations.

\section{Mapping the initial states of clusters as stellar dynamical systems}

We now turn to the question of the early evolution of star clusters from the point at which they become predominantly stellar dynamical systems. Below we review recent work that uses N-body calculations to map the state of observed young clusters back to this point.

\subsection{Morphology}

Young star clusters are often modestly flattened (axis ratio on the plane of the sky > 0.7; Frenk and Fall 1982, O'Connell et al. 1994). Such morphologies are suggestive of a dynamical trigger for cluster formation such as cloud-cloud collisions. However, collisions between homogeneous clouds should give rise to clusters that are extremely flattened - for example, the collision between two clouds that can cool to $10 \mathrm{~K}$ should form 'pancake' structures of aspect ratio $\sim 10^{-4}$ if they collide head on at a relative velocity of $20 \mathrm{~km} / \mathrm{s}$. The question therefore arises: can stellar dynamical processes puff up such razor thin initial conditions so that after a few crossing times they resemble the observed states of young clusters?

Early simulations on the dynamical evolution of flattened systems (Aarseth and Binney 1978) suggested that such rapid puffing up, and erasure of the initial morphology, could indeed occur. A re-investigation of this issue (Boily, Clarke and Murray 1999) showed that this is the case only where the stars are initially distributed in a mono-layer (i.e., with scale height, $H$, less than the mean interstellar separation $l_{*}$ ), in which case it is well known that two-body relaxation puffs up the distribution on a crossing time (Rybicki 1972). Boily et al. showed that in the opposite case $\left(H>l_{*}\right)$, the morphological evolution is controlled by the violent relaxation of the cluster, and in this case adiabatic invariance can be used to simply relate the initial shape to its relaxed morphology. Application of this mapping to the LMC globular clusters suggests they are unlikely to have 
arisen from initial structures that were flattened by more than the ratio $5: 1$. Murray et al. 1999 concluded that this conclusion was not radically affected if the flattened system initially comprised dissipative clumps rather than stars.

Similar N-body simulations led Goodwin (1998) to question whether the LMC globular clusters could indeed have arisen from cloud-cloud collisions, given the very flattened initial states expected in this case. However, this is unlikely to be an issue, given the very inhomogeneous nature of GMCs. If the colliding clouds are clumpy, then the shocked layer is strongly buckled due to the net momentum carried across the symmetry plane (see, e.g., Kimura and Tosa 1996). Indeed, for clumpy cloud collisions, the morphological imprint may be counterintuitive: in the case of colliding clouds with rather a low filling factor, there is considerable interpenetration of the clouds and the resulting structure is prolate with its long axis along the direction of the collision (Gittins, private communication). This example cautions against the use of the position angle of a flattened cluster to deduce the direction of the triggering agent.

\subsection{Substructure}

The ISM appears to be organised as a hierarchy of clumpy density structure and it is thus natural to ask whether, during cluster formation, the stellar distributions reflect this sort of nested structure. If so, then the dominant interactions may be experienced by young stars at the highest density, least populous, level of the clustering hierarchy: it might then be unimportant whether such small scale groupings are themselves organised into larger scale groupings that have the potential to survive as bound clusters. In this case, one might not expect any significant differences between the properties of stellar populations in clusters or in the field. An alternative scenario would be that as large scale clusters are formed, the clumpy gas is swept up and homogenised, so that stars forming in this environment know only about the large scale potential of the parent cluster.

The obvious way to address this question is just to look at the spatial distributions of stars in young clusters and decide on this basis whether the distributions are homogeneous or highly sub-clustered. The problem here is that although a cluster may be young (in the sense of being only a few of its own crossing times old) it may be plenty old enough for a memory of substructure to have been erased. Evidently one can pursue this by looking at clusters at the youngest possible ages, although here patchy extinction might mimic the effect of sub-clustering. Alternatively, one can examine slightly older clusters, where the stellar census is reasonably complete, and use N-body simulations to constrain how sub-clustered the initial conditions could have been. Below, we outline some case studies.

In the Orion Nebula Cluster (ONC), the observed stellar distribution is very smooth, even though the cluster (at an age of $2 \mathrm{Myr}$, i.e., several crossing times) is extremely youthful. Indeed, an analysis of clustering statistics in the ONC showed that its present state is incompatible with any significant substructure ( Bate et al. 1998). At face value, this would appear to strongly support the notion of smooth initial conditions. However, Scally and Clarke (2001) showed that a variety of hierarchically sub-clustered initial conditions are compatible with the observed state of the cluster at its present age: the dissolution timescale in the 
high density sub-clusters is so short that a smooth relaxed structure can be readily obtained within a few Myr.

In the LMC globular clusters, there is more positive evidence for an initially subclustered stellar distribution: as noted by Elson (1991), clusters younger than $\sim 40$ Myr exhibit lumpy light profiles, whereas older clusters are rather smooth. Goodwin (1998) used this data to constrain the allowed parameter space for sub-clustering in the initial conditions and found that a range of possible configurations were allowed. It was found however that a relatively large number of initial sub-clusters $(>200)$ are required in order to avoid a highly cusped density distribution.

\subsection{Mass segregation.}

There are a number of instances of young clusters that show evidence for mass segregation. One explanation is that such clusters are mass segregated at birth, as would be the case if competitive accretion were important (see 2.2 above). However, massive stars will also congregate in the central regions of clusters through two-body relaxation. $\mathrm{N}$-body simulations are often required in order to settle whether the state of observed young clusters requires primordial mass segregation.

The best studied cluster in this regard is the ONC, where Bonnell and Davies (1998) showed that the observed concentration of massive stars towards the cluster core is incompatible with two-body relaxation: specifically, they showed that the central Trapezium of massive stars can be formed within the required timescale only if the 30 most massive stars are initially concentrated within a radius containing $<20 \%$ of the cluster mass. This result argues strongly for primordial mass segregation.

The dense cluster R136 in the core of 30 Dor also shows evidence for mass segregation (Sirianni et al. 2000). Here Portegies-Zwart et al. 1998 argued that this can be explained by two-body relaxation over the cluster lifetime and demonstrated that stellar collisions within the resulting core of massive stars can produce a number of blue stragglers in this region. In the case of the LMC globular clusters, colour gradients argue for a degree of mass segregation (Fischer et al. 1998; Kontizas et al. 1998), which cannot be explained by two-body relaxation (Boily et al. 1999).

In summary, then, it would seem that although two-body effects will undeniably enhance the population of high mass stars in cluster cores, there are at least some systems in which there is good evidence that mass segregation is primordial.

\subsection{Summary}

To summarise the results of the above sections, it would appear that clusters are mildly flattened, possibly clumpy, and already mass segregated at the point that the stars become the majority mass component. 


\section{The return of dark matter to globular cluster formation?}

We now briefly address the issue of how protocluster gas is assembled. We have already argued (see 2.1 above) that the requirement that the gas be highly inhomogeneous argues against an identification of the cluster mass scale with a thermal Jeans mass, since then the gas would have passed through a phase where thermal pressure would have smoothed out any pre-existing density structure. This would seem to imply that the gas in a proto-cluster cloud is assembled by some mechanism other than its own self-gravity. Such mechanisms are seen in normal galaxies where GMCs are assembled through the potential of the spiral arms, and, more spectacularly in the case of colliding galaxies.

Recently, Bromm and Clarke (in prep.) have investigated how protocluster gas might be assembled during the formation of a dwarf galaxy. The dark matter halo of such a galaxy contains substructure imparted by the Cold Dark Matter fluctuation spectrum, so that as this halo approaches virialisation, it contains a number of dark matter mini-potential wells. Baryons in these miniwells collapse dissipatively and form compact self-gravitating structures. During the subsequent virialisation of the halo, these dark mini-wells are erased by violent relaxation, whereas the baryons are compact enough to be unscathed. As a result, dense self-gravitating baryonic cores (with typical masses in the range $10^{5}-10^{6} M_{\odot}$ ) are formed during the virialisation of the parent dwarf.

It is tempting to identify such concentrations with proto-globular clusters, as they have the right masses and sizes. Current simulations cannot resolve the sub-fragmentation of these baryonic concentrations, so can't rule out that they might alternatively collapse to form super-massive black holes. However, the Jeans mass in the gas is low, thus raising the possibility of sub-fragmentation.

Do such simulations re-open the possibility that dark matter is involved in the formation of old globular clusters? This scenario (see also Peebles 1984, Rosenblatt et al. 1988) has fallen into disfavour following the demonstration that globular clusters are not surrounded by massive dark halos (Moore 1996). However, the current simulations demonstrate that such halos would be stripped off during the virialisation of the parent halo: the resulting 'globular cluster' should contain no dynamical clues of any previous role for dark matter. In such simulations, the role of the dark matter is simply to provide the potential well within which the baryons are gathered up and compressed. (Note that the selfgravity of the gas plays no role in its initial assembly, since the mini-wells are dark matter dominated in these initial stages).

Fortuitously or not, the simulations produce around 5 'globular clusters' within each dwarf galaxy, a number that places them at the upper end of the observed globular cluster content for dwarf spheroidal galaxies (it is of course easy to invoke survival arguments to reduce this number if desired). In a hierarchical cosmology, many of these dwarf galaxies and their attendant globular clusters would end up being incorporated in more massive systems, as advocated by Cote et al. 1998. It would be tempting to identify the population of metal poor globular clusters in galaxies as originating from such acts of galactic cannibalism, and to invoke the mechanism described here to explain why globular clusters might be readily produced during dwarf galaxy formation. 
Acknowledgments. I am indebted to Matthew Bate, Ian Bonnell, Volker Bromm and Dave Gittins for helpful discussions during the writing of this paper.

\section{References}

Aarseth, S., \& Binney, J. 1978, MNRAS, 185, 227

Bate, M.R., \& Burkert, A. 1997, MNRAS, 288,1060

Bate, M.R., Clarke, C.J., \& McCaughrean, M.J. 1998, MNRAS, 297,1163

Boily, C.M., Clarke, C.J., \& Murray, S.D. 1999, MNRAS, 302, 399

Bonnell, I., Bate, M., Clarke, C., \& Pringle, J. 2001a, MNRAS, 323, 785

Bonnell, I., Clarke, C., Bate, M., \& Pringle, J. 2001b, MNRAS, 324, 573

Bonnell, I.A., \& Davies, M.B. 1998, MNRAS, 295, 691

Cote, P., Marzke, R.O., \& West, M.J. 1998, ApJ 501, 554

Elson, R.A.W., 1991, ApJS, 76, 185

Elmegreen, B.G., \& Efremov, Y.N. 1997, ApJ, 480, 235

Fall, S.M., \& Rees, M.J. 1985, ApJ, 298, 18

Fischer, P., Pryor, C., Murray, S., Mateo, M., \& Richtler, T. 1998, AJ, 115, 592

Frenk,C.S., \& Fall, S.M. 1982, MNRAS, 199, 565

Goodwin, S.P. 1997, MNRAS, 284, 785

Goodwin, S.P. 1998, MNRAS, 294, 47

Harris, W., \& Pudritz, R. 1994, ApJ, 429,177

Hoyle, F. 1953, ApJ, 118, 513

Hunter, C. 1962, ApJ, 136, 594

Kimura, T., \& Tosa, M. 1996, A\&A, 308, 979

Klessen, R., Burkert, A., \& Bate, M.R. 1988, ApJ, 501, L205

Kontizas, M., Hatzidimitriou, D., Bellas-Velidis, I., Gouliermis, D., Kontizas, E., \& Cannon, R.D. 1998, A\&A, 336, 503

Lada, C.J., Margulis, M., \& Dearborn, D. 1984, ApJ, 285, 141

Larson, R.B. 1978, MNRAS, 184, 69

Lubow, S.H., \& Pringle, J.E. 1993, MNRAS, 263, 701

McLaughlin, D.E., \& Pudritz, R.E. 1996, ApJ 457, 578

Murray, S.D., \& Lin, D.N.C. 1989, ApJ, 339, 933

Murray, S.D., \& Lin, D.N.C. 1996, ApJ, 467, 728

Murray, S.D., Raymondson, D.A., \& Urbanski, R.A. 1999, ApJ, 517, 829

O'Connell, R.W., Gallagher, J.S., \& Hunter, D.A. 1994, ApJ, 433, 65

Padoan, P., Jimenez, R., \& Jones, B. 1977, MNRAS, 285, 711

Peebles, P.J.E. 1984, ApJ, 277, 470

Portegies Zwart, S., Makino, J., McMillan, S.L., \& Hut, P. 2001, in Stellar Collisions, Mergers and their Consequences, ed. M. Shara, ASP Conf. Ser.

Rees, M.J., \& Ostriker, J.P. 1977, MNRAS, 179, 541

Rosenblatt, E.I., Faber, S.M., \& Blumenthal, G.R. 1988, ApJ, 330, 191 
Rybicki, G.B. 1972 in IAU Colloq. 10, Gravitational N-Body Problem ed. M. Lecar (Reidel), 22

Scally, A., \& Clarke, C. 2001, MNRAS, 325, 449

Sirianni, M., Nota, A., Leitherer, C., De Marchi, G., \& Clampin, M. 2000, ApJ 533,203

Zinnecker, H. 1982, in Symposium on the Orion Nebula to Honour Henry Draper, eds. A. E. Glassgold et al., New York Academy of Sciences, 226

\section{Discussion}

G. Piotto: Can you comment on the number of binaries that are formed and where they are formed?

C. Clarke: These simulations typically form plenty of binaries. Bate et al. are planning a detailed investigation of this question.

J. Gallagher: Do you have any insights into how your models would predict scaling with cluster mass? In particular, is there a physical reason why the cluster sequence should show a wide range in mass $\left(>10^{3}\right)$ for a given physical scale (half-mass radius for example)? Observationally, this shows up as a minimum scale for massive clusters of a few parsecs.

C. Clarke: I have tried to argue that the mass of a cluster does not have anything to do with the Jeans mass (as it need not, if it's not the cluster's selfgravity that is assembling it). In this case, one does not expect any particular correlations between the masses and sizes of clusters, except, of course, by those imposed by 'survival triangle' type arguments.

H. Zinnecker: We seem to see the formation of massive star clusters in interacting and merging starburst galaxies, such as the Antennae. The process that you described (involving dark matter) is different. Can you synthesise the two scenarios?

C. Clarke: I've been advocating that cluster formation involves the sweeping up of gas by some mechanism that is more than its self-gravity. Clearly galaxy interactions provide an excellent mechanism for this. All I am doing here is to point out that during the process of dwarf galaxy formation, inhomogeneities in the dark matter distribution can play a similar role. 